\title{
Modelling Co-movement between Onshore and Offshore RMB Exchange Rate Based on Copula
}

\author{
J.Z. Du \\ Department of Management Sciences \\ City University of Hong Kong \\ Hong Kong
}

\author{
K.K. Lai \\ International Business School \\ Shaanxi Normal University \\ Xian, China \\ Department of Management Sciences \\ City University of Hong Kong \\ Hong Kong
}

\begin{abstract}
This paper investigates the dependence of the exchange rate of Onshore RMB and Offshore RMB against U.S. dollar, i.e. CNY and CNH, based on copula models. We select ten different copulas to construct multivariate distribution for RMB exchange rate. The empirical results show that time-invariant Student-t copula is the best model to fit the sample data. The positive of upper and lower dependence indicates that $\mathrm{CNY}$ and $\mathrm{CNH}$ series tend to move in the same direction. Also, the results indicate that the dependence between the two exchange rates is time-invariant and symmetric, which means that traditional models such as Pearson's correlation are inappropriate to measure the correlations between these markets.
\end{abstract}

\section{Keywords-copula; RMB exchange rate; dependence modelling}

\section{INTRODUCTION}

The foreign exchange market is of great value to the country's economy as well as the industry. The exchange rate of RMB plays an important role in determining China's economic prospects. For facilitating RMB internationalization, China is developing the RMB offshore business in Hong Kong where RMB has become convertible. The financial markets have distinguished between onshore and offshore RMB as $\mathrm{CNY}$ and CNH, respectively. These two rates are determined by the economic fundamentals of China as well as other countries. However, the demand for RMB in the two different markets leads to the difference between the two rates since $\mathrm{CNH}$ is more exposed to international markets. Also, the difference between interest rates in these two markets and business activities trigger capital flows between the two markets, creating the co-movement of exchange rates.

The copula is introduced to flexibly describe the dependence between variables. Since Sklar (1959) first proposed the Sklar theorem, the copula has gained rapid development in financial time series [1]. The theorem shows that all n-dimensional joint distribution functions can be decomposed into $\mathrm{n}$ marginal distributions and a copula. The $\mathrm{n}$ marginal distributions describe location, scale and shape parameters of a variable, i.e. mean, standard deviation, skewness and kurtosis and the copula sufficiently describes the dependence among the $n$ variables. Based on the Sklar theorem, Hutchinson and Lai (1990), Joe (1997) and Nelsen (1999) extended the copula theorem further [2-4]. Embrechts (1999) introduced the concept into finance literature [5]. Copula has already been widely used in various fields of finance, such as risk management, asset pricing, option valuation and so on. However, the initial researchers only considered the unconditional case and just focused upon the constant scenarios, ignoring the time-varying case in particular. Many researchers have reviewed the constant copula applications such as $\mathrm{Li}$ (2000), Cherubini, et al.(2004) and Yan (2007) [6-8].

This paper expects to make several contributions. First, we introduce the copula to the field of foreign exchange market in a developing country and investigate the dependence between two different exchange rates of the same currency in different markets. The second contribution is to compare time-varying copula model and time-invariant model with marginal distributions fitted by the GARCH model with normal, student$\mathrm{t}$ and skewed student-t innovations to measure the time-varying dependence. It is found that the strong persistence, in the dynamics of the dependence, reflects fluctuations in the dependence parameter, over the sample period. Thirdly, we investigate the relationship between $\mathrm{CNY}$ and $\mathrm{CNH}$ over the same period, and compare the difference.

In this paper. Section 2 outlines the copula and marginal models. In Section 3, we conduct the empirical experiment. Section 4 concludes the paper.

\section{MODEL SPECIFICATION AND ESTIMATION}

\section{A. The Copula Function}

The copula function is proposed to measure the dependence of multivariate time series. The copula based multivariate model allows study of the marginal model separately from dependence structure, making it more flexible to specify the model.

To better learn the copula theory, let's start with the Sklar Theorem. Sklar (1959) stated that a two dimensional joint distribution can be decomposed into $\mathrm{n}$ univariate marginal distributions and a dependence structure called copula (two dimensional). Given two continuous random variables $\mathrm{X}$ and $\mathrm{Y}$, the joint distribution is:

$$
F_{X Y}(x, y)=C\left(F_{X}(x), F_{Y}(y)\right)
$$


where $F_{X}(x)$ and $F_{Y}(y)$ are the marginal distributions of $\mathrm{X}$ and $\mathrm{Y}$, respectively, while $F_{X Y}(x, y)$ is the joint distribution. Set $\mathrm{U}$ and $\mathrm{V}$ as probability integral transform (PIT) of variable $\mathrm{X}$ and $\mathrm{Y}$, respectively, then $U=F_{X}(X), V=F_{Y}(Y)$. According to the construction, $C\left(F_{X}(x), F_{Y}(y)\right)$ is a bivariate cumulative distribution function (c.d.f.) with marginal distribution Unif $(0,1)$.

The copula $C(u, v)$ capture the dependence structure to map the margins $F_{X}(x)$ and $F_{Y}(y)$ to the joint distribution $F_{X Y}(x, y)$. If the margins $F_{X}$ and $F_{Y}$ are continuous, then $C$ is uniquely determined on $\operatorname{RanF}_{X} \times \operatorname{RanF}_{Y}$. What makes Equation (1) particularly meaningful is the converse of the Sklar theorem: given any copula $C$ and two univariate distributions $F_{X}(x)$ and $F_{Y}(y)$, a multivariate distribution $F_{X Y}(x, y)$ with marginal distribution $F_{X}(x)$ and $F_{Y}(y)$ is determined by Equation (1).

\section{B. Marginal Distribution Model}

In order to build the model for bivariate distribution with copula, the marginal distribution for the single series must be specified first. Is has been commonly accepted that financial time series returns always exhibit several stylized characteristics like fat tails, volatility clustering and conditional heteroskedasticity (Mohammadi and Su, 2010) [9]. One of the most popular methods to model an individual financial variable is the GARCH family model, proposed by Engle (1982), Bollerslev (1986), which has been widely applied in the financial area by McNeil and Frey (2000) and Ning (2010) [1013]. It has been proved that fitting the financial time series with GARCH can capture most of its features, including volatility clustering, autoregression in mean, skewness and kurtosis. To overcome the inability of GARCH to model asymmetric volatility, it has been extended and generalized to EARCH, GJR-GARCH, FIGARCH and so on. These extended models enhance the performance of the original GARCH model introduced by Engle and can fit the financial time series more flexibly. Among these models, GJR-GARCH model is most widely used and can consider the asymmetric volatility and the leverage effect after markets experience shocks. Inspired by the results of pioneer researchers, we combine ARMA (m,n) with the GJR-GARCH $(p, q)$ model to model the daily exchange rate returns in this paper:

$$
\begin{aligned}
& r_{t}=\phi_{0}+\sum_{j=1}^{m} \phi_{j} r_{t-j}+\varepsilon_{t}-\sum_{i=1}^{n} \theta_{i} \varepsilon_{t-i} \\
& h_{t}=\omega_{0}+\sum_{i=1}^{p} \beta_{i} h_{t-i}+\sum_{j=1}^{q} \alpha_{j} \varepsilon_{t-j}^{2}+\sum_{j=1}^{q} \gamma_{j} \varepsilon_{t-1}^{2} I_{t-j}
\end{aligned}
$$

$$
\varepsilon_{t}=\eta_{t} \sqrt{h_{t}} \eta_{t} \sim f(0,1) \text { i.i.d. }
$$

where $\mathrm{m}, \mathrm{n}, \mathrm{p}$ and $\mathrm{q}$ are positive integers. These four orders are determined by empirically finding the optimal model with Akaike Information Criterion (AIC). $\phi_{j}$ and $\theta_{j}$ are the parameters for autoregressive (AR) and moving average (MA) parameters, respectively. $\varepsilon_{t}$ is the residual of the process, satisfying $E\left(\varepsilon_{t}\right)=0$ and $E\left(\varepsilon_{t} \varepsilon_{s}\right)=0$ with $s \neq t \cdot h_{t}$ is the conditional variance of the residual $\varepsilon_{t} \cdot \eta_{t}$ is the standard residual of the process with zero mean and unit variance. $I_{t}$ is an indicator, equal to 1 if $\varepsilon_{t-1} \leq 0$ and 0 otherwise. The indicator makes it possible to model the asymmetric effects of good and bad news. $\alpha+\gamma$ reveals the impact of bad news and $\alpha$ is the impact of good news. If $\gamma \neq 0$, there exists the leverage effect, otherwise, the leverage effect does not exist and the GJR-GARCH model is reduced to the original GARCH model.

The standard residual $\eta_{t}$ can be fitted by several parametric distributions including Normal, Student-t, Skewed-t distribution and so on. In this paper, we follow Patton (2012) to choose the flexible and simple Skewed-t distribution of Hansen. Although the excess kurtosis as stated can be captured by the Student-t distribution, it is inappropriate for modelling skewness.

\section{DATA AND EMPIRICAL RESULTS}

\section{A. Data Description}

To figure out the dependence structure of spot prices, we use daily returns of the exchange rate of CNY and CNH against USD from August 24, 2010 to September 11, 2014, making a total of 1048 observed data series, all from Bloomberg.

Table 1 summarizes statistics of daily returns of RMB exchange rate. Skewness of all the returns is positive, suggesting that there is a greater probability of increases in returns. The high kurtosis of the returns reveals that extreme value changes often occur when the tail of return distributions shows fatness.

TABLE I. STATISTICS OF DAILY RETURN OF CNY AND CNH

\begin{tabular}{cll}
\hline & CNY & CNH \\
\hline Mean & -0.0134 & -0.0122 \\
Max. & 0.4784 & 1.3214 \\
Min. & -0.5554 & -0.8996 \\
St.D & 0.1091 & 0.1609 \\
Skewness & -0.1787 & 1.1623 \\
Kurtosis & 6.1351 & 16.8070 \\
J-B & 326.0791 & 6420.2 \\
ADF & -30.2556 & -28.3170 \\
ARCH-LM Test & 21.7235 & 102.3464 \\
\hline
\end{tabular}




\section{B. Results for Marginal Distribution}

In this section, the ARMA(m, n)-GJR(p, q) type models for each return series are estimated. We choose the most suitable model with the criteria of BIC values by considering the value of parameters $\mathrm{m}, \mathrm{n}, \mathrm{p}$ and $\mathrm{q}$ ranging from zero up to the maximum lag of two. All the parameters estimates of marginal distributions are included in Table 2 which summarizes the best model for the two marginal distributions: the best model for the marginal CNY and CNH is $\operatorname{ARMA}(0,0)-G J R(1,1)$.

TABLEII. PARAMETERS ESTIMATION FOR MARGINAL DISTRIBUTION

\begin{tabular}{cll}
\hline & CNY & CNH \\
\hline $\begin{array}{c}\text { Mean equation } \\
\phi_{0}\end{array}$ & -0.0099 & -0.0088 \\
Variance & & \\
Equation & & \\
$\omega_{0}$ & 0.0003 & 0.0004 \\
$\beta_{1} \cdot$ & 0.1514 & 0.1369 \\
$\alpha_{1}$ & 0.8404 & 0.8571 \\
Tail & 4.6755 & 4.2563 \\
Ljung-Box(10) & 5.8876 & 11.3696 \\
& 0.8246 & 0.3295 \\
ARCH-LM(10) & 2.8157 & 6.1175 \\
& 0.7284 & 0.8053 \\
\hline
\end{tabular}

\section{Results for Copula Models}

From Table 3, it is obvious that the Student-t copula performs best for the pair $\mathrm{CNY}$ and $\mathrm{CNH}$, according to the AIC criteria. The degrees freedom of Student-t copula are relatively low (ranging from 4 to 7), meaning that the co-movement and tail dependence of all the pairs are non-normal. The calculated tail dependence value for the pair is 0.584 . We also find that values of parameters for Gaussian and Student-t copulas are all positive and very close to the linear correlation coefficient for each pair of series.

Although the parameters of Clayton, Rotated Clayton, Gumbel and Rotated Gumbel copulas reflect the weak dependence of the pairs, the values are significant. The lower tail dependence is 0.841 . For the Rotated Gumbel copula, value of lower tail dependence is 0.897 . To measure the asymmetric upper tail dependence, the Rotated Clayton and Gumbel copulas are conducted. The values of upper tail dependence of the pair are 1.588 for Rotated Clayton copula and 1.574 for Gumbel copula. Moreover, the SJC copula, which can measure asymmetric upper and lower tail dependence, has also been estimated. The null hypothesis of $\lambda^{U}=\lambda^{L}$ is rejected, meaning symmetric dependence. However, the symmetric Student-t copula is selected to be the most appropriate model among the seven time-invariant copulas to measure the dependence between $\mathrm{CNY}$ and $\mathrm{CNH}$.

Panel B of Table3 shows estimations of the time-varying Gaussian, Rotated Gumbel and SJC copula. According to the AIC, the time-varying Gaussian, Rotated Gumbel and SJC copula can improve the performance of the relatively timeinvariant copulas. However, the performance of dynamic copulas can not exceed that of the time-invariant Student-t copula based on the AIC criteria.

TABLE III.ESTIMATION FOR THE COPULA MODELS

\begin{tabular}{|c|c|c|}
\hline & & CNY-CNH \\
\hline \multicolumn{3}{|l|}{$\begin{array}{l}\text { Panel A: time- } \\
\text { invariant }\end{array}$} \\
\hline \multirow[t]{2}{*}{ Gaussian copula } & $\rho$ & 0.584 \\
\hline & AIC & -2274.1 \\
\hline \multirow[t]{3}{*}{ Student-t copula } & $\rho$ & 0.584 \\
\hline & $v$ & 14.084 \\
\hline & AIC & -2459.2 \\
\hline \multirow[t]{2}{*}{ Clayton copula } & $\alpha$ & 0.841 \\
\hline & AIC & -1962.5 \\
\hline \multirow[t]{2}{*}{$\begin{array}{c}\text { Rotated Clayton } \\
\text { copula }\end{array}$} & $\alpha$ & 0.897 \\
\hline & AIC & -1650.8 \\
\hline \multirow[t]{2}{*}{ Gumbel copula } & $\delta$ & 1.588 \\
\hline & AIC & -2175.4 \\
\hline \multirow[t]{2}{*}{$\begin{array}{c}\text { Rotated Gumbel } \\
\text { copula }\end{array}$} & $\delta$ & 1.574 \\
\hline & AIC & -2349.5 \\
\hline \multirow[t]{3}{*}{$\begin{array}{l}\text { Symmetrized Joe- } \\
\text { Clayton }\end{array}$} & $\lambda^{U}$ & 0.346 \\
\hline & $\lambda^{L}$ & 0.389 \\
\hline & AIC & -2248.7 \\
\hline \multicolumn{3}{|l|}{ Panel B: time-varying } \\
\hline \multirow[t]{4}{*}{ TVP Gaussian copula } & $\omega_{N}$ & 0.997 \\
\hline & $\alpha_{N}$ & -0.324 \\
\hline & $\beta_{N}$ & 1.658 \\
\hline & AIC & -2358.2 \\
\hline \multirow[t]{4}{*}{ TVP Rotated Gumbel } & $\omega_{T}$ & 1.894 \\
\hline & $\alpha_{T}$ & -0.135 \\
\hline & $\beta_{T}$ & -3.362 \\
\hline & AIC & -2440.9 \\
\hline \multirow[t]{7}{*}{ TVP SJC copula } & $\omega_{U}$ & 3.356 \\
\hline & $\alpha_{U}$ & -19.962 \\
\hline & $\beta_{U}$ & -1.094 \\
\hline & $\omega_{L}$ & 2.103 \\
\hline & $\alpha_{L}$ & -3.222 \\
\hline & $\beta_{L}$ & -2.034 \\
\hline & AIC & -2394.0 \\
\hline
\end{tabular}

\section{CONCLUSION}

In this paper we have combined constantor time-varying copulas with GARCH-type models to construct the joint conditional multivariate distribution for the RMB exchange rate series. One of our main findings is that the constant Student-t copula is a good compromise for effectively fitting the dependence structure between On-Shore and Off-Shore RMB exchange rate. The parameters of Student-t copula can be 
time-varying, but the dynamics of parameters improve little of forecasting performance.

These findings have significant implications for risk management and asset pricing in RMB exchange markets. For instance, the existence of both positive upper and lower tail dependence indicates much higher risk than the case of no tail dependence. Furthermore, the range of tail dependence is vital to risk-averse investors, who dislike risk and prefer to stay away from high risk investment in their portfolios.

\section{ACKNOWLEDGMENTS}

This work is supported by the Strategic Research Grant of City University of Hong Kong (no. 7004268)

\section{REFERENCES}

[1] Sklar, A. Fonctions de repartition à n dimensions et leurs marges. Publications de l'Institut de Statistique de l'Université de Paris 8, 229231.1959.

[2] Hutchinson, T. P., and Lai, C. D. Continuous bivariate distributions, emphasising applications. Rumbsy Scientific Publishing, Adelaide.1990.

[3] Joe, H. Multivariate Models and Dependence Concepts. Chapman and Hall, London.

[4] Nelsen, R.B. 1999. An Introduction to Copula. Springer, New York.1997.

[5] Embrechts, P., McNeil, A.J., and Straumann, D. Correlation and dependency in riskmanagement: properties and pitfalls. In: Dempster, M., and Moffatt, H. (Eds), Risk Management: Value at Risk and beyond. Cambridge University Press, 2002.

[6] Li, D.X. On default correlation: a copula approach. The Journal of Fixed Income 9, 43-54. 2000.

[7] Cherubini, U., Luciano, E., and Vecchiato, W. Copula Methods in Finance. John Wiley \& Sons, England.2004.

[8] Yan, J. Enjoy the joy of copulas: with a package copula. Journal of Statistical Software 21, 1-21.2007.

[9] Mohammadi, Hassan and Lixian Su. "International Evidence on Crude Oil Price Dynamics: Applications of Arima-Garch Models." Energy Economics 32, no. 5: 1001-1008.2010

[10] Engle, Robert F. "Autoregressive Conditional Heteroscedasticity with Estimates of the Variance of United Kingdom Inflation." Econometrica 50, no. 4. 987-1007.1982.

[11] Bollerslev, Tim. "Generalized Autoregressive Conditional Heteroskedasticity." Journal of Econometrics 31, no. 3.307-327. 1986.

[12] McNeil, Alexander J and Rüdiger Frey. "Estimation of Tail-Related Risk Measures for Heteroscedastic Financial Time Series: An Extreme Value Approach." Journal of empirical finance 7, no. 3. 271-300. 2000.

[13] Ning, Cathy. "Dependence Structure between the Equity Market and the Foreign Exchange Market-a Copula Approach." Journal of International Money and Finance 29, no. 5. 743-759. 2010. 